\title{
ESTUDO DE TEMPO E MOVIMENTO NO PROCESSO DE FABRICAÇÃO EM UMA EMPRESA DE XAROPE DE GUARANÁ
}

\author{
Alexandre Cunha Marquezine - alexandremarquezine@gmail.com1 \\ Patrícia Werneck S. de Oliveira - paticso@yahoo.com.br2
}

\begin{abstract}
RESUMO: Este trabalho descreve a importância do estudo de Cronoanálise no setor produtivo, visando a melhorias no processo de produção de xarope de guaraná. Tal abordagem se faz necessária pois a partir deste é possível identificar quais os fatores são responsáveis pelo atraso na linha de produção. O objetivo deste trabalho é verificar o nível de produtividade da empresa a fim de buscar o método mais eficiente de maximizar o setor de produção do xarope de guaraná. Esta tarefa foi obtida mediante o estudo de caso a partir do método de Cronoanálise, verificou- se todo o processo de fabricação do xarope de guaraná em uma empresa de pequeno porte da cidade de Cataguases, Minas Gerais. A pesquisa demonstrou que em média um trabalhador produz em média 119 bombonas de xarope de guaraná, porém alguns fatores prejudiciais na produção deveriam ser corrigidos para haver uma melhoria na produtividade, tal como, layout da área de produção, no qual, ocasiona congestionamento de funcionários no corredor da área de produção em determinado momento do dia. Palavra - chave (s): Cronoanálise; Xarope de guaraná; Processo de produção.
\end{abstract}

Keyword (s): Chronoanalysis; Guarana syrup; Production process.

\section{INTRODUÇÃO}

O Mercado do guaraná tem aumentado nos últimos anos, de acordo com a folha de São Paulo, o concentrado obteve um crescimento superior a dezoito por cento em 2002, porém teve uma queda na força até chegar uma baixa de seis por cento em 2008 e agora novamente se acelera.

Segundo Camargo et al. (2006), A produção do guaraná brasileiro é quase toda consumida pelo mercado, avalia que cerca de setenta por cento da produção deste seja utilizada pelos fabricantes de refrigerante, entretanto o restante é vendido em forma de xarope, extrato, pó, bastão e outros subprodutos, pequena parte são exportadas.

De acordo com Ribeiro (2012, apud Tfounir et al., 2007). O guaraná vem sendo utilizado na região Norte e em diversos estados brasileiros como fonte energético devido seus compostos e nas industrias fármaco exercendo a função em vários medicamentos como o princípio ativo.

O trabalho tem como objetivo a realização do estudo de Cronoanálise em uma empresa do ramo Alimentício. Cuja finalidade é a produção do Xarope de Guaraná e granola. Desta maneira procura-se melhorar, padronizar, e minimizar o tempo de produção do xarope de guaraná, visando o aumento de sua produção. Com os dados adquiridos através de visitas, foram feitas cronometragens, realizando uma análise de tempo real e sintético. Nesse intuito, efetuou-se um estudo em uma empresa da zona da mata mineira, localizada na cidade de Cataguases, aplicando a ferramenta de cronoanálise na busca de melhoria nos processos, bem como o estudo de tempo de algumas operações de modo a realizar uma padronização dos tempos dos operários.

\section{METODOLOGIA}

A metodologia de acordo com Gill (1999) é a linha de raciocínio adotada no processo de pesquisa. Foram realizadas pesquisas bibliográficas por meio de livros e internet, também foram consultados artigos científicos. Todas as buscas foram efetuadas no período, entre 13 de março a 30 de maio de 2017. A escolha do Artigo foi feita em semelhança ao tema proposto. Para a realização do estudo de caso foram feitas visitas a empresa estudada, em que o processo de estudo investigado foi a produção do xarope de guaraná. Foram cronometrados tempo do processo e o 
movimento dos operadores foi observado. Diante dos conhecimentos adquiridos na visita a empresa, o estudo sobre os métodos de cronoanálise foram obtidos. Realizou-se assim, cálculo da capacidade produtiva e gráfico de controle. A construção do gráfico de controle foi feita através do software Minitab 17.

\subsection{Empresa Objeto de Estudo}

Realizado em uma empresa da Zona da Mata Mineira, localizada em Cataguases, Minas Gerais, com fim específico na produção do xarope de guaraná, possui sete operários na área da produção, são produzidos certa de cerca de dezoito mil bombonas por mês, porém dependendo da época do ano sua produção pode oscilar para mais ou para menos, como pode ser observado na Figura 1.

Figura 1: Gráfico de vendas das bombonas de cinco litros de xarope de guaraná vendidos no ano de 2016

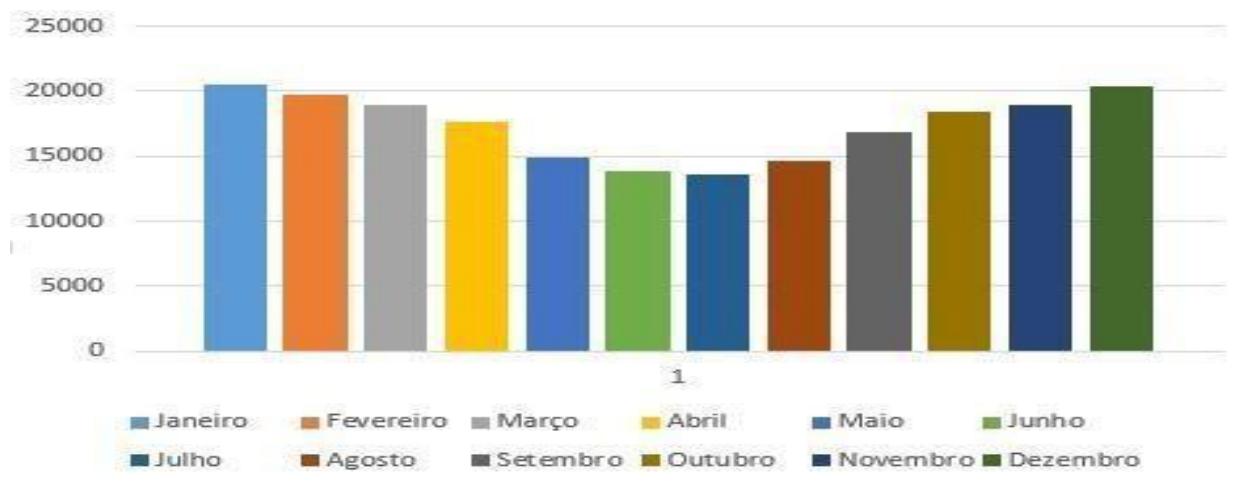

Fonte: Autor. Ano 2017

\subsection{Xarope de Guaraná}

De acordo com o Decreto ${ }^{\circ} 6.871$, de 4 de junho de 2019, pronuncia que o xarope é o produto, ou seja, a bebida, não gaseificado, adquirido pela dissolução, em água potável, de parte do vegetal, suco da fruta ou polpa e açucar, em uma concentração com pelo menos cinquenta e dois por cento de açucares, dado em peso, preparado a vinte graus Celsius (BRASIL, 2009).

É feito de uma fruta tipicamente brasileira, o guaraná. O xarope de guaraná traz diversos benefícios a saúde pelas suas propriedades energéticas aumentando a capacidade cerebral com o aumento significativo de memória e concentração. É utilizado para o preparo de açaí pois possui alto teor de açúcar e boa viscosidade abaixo da temperatura, seu aroma realça o sabor e garante o maior rendimento.

\section{REVISÃO DE LITERATURA}

\subsection{Cronoanalise}

Para Lima (2016), A cronoanalise é frequentemente usada nos estudos de temos. Ela é identificada pelo uso de cronometragem para obtenção do tempo padrão de uma operação. É uma técnica usual em ambientes industriais com serviços repetitivos, sendo utilizado para determinar custos da mão de obra, capacidade produtiva, metas de produção.

\subsection{Tempo e Movimento}

O estudo de tempo e movimento tem como objetivo segundo Duarte (2015): 
(1) Estabelecer requisitos e padrões do cargo ou da função; (2) levantamento e determinação precisos da quantidade necessária de tempo a partir da avaliação comparativa em relação em relação ao padrão anteriormente, estabelecido da capacidade ou do esforço realizado pelo trabalhador objetivando a execução de uma tarefa especifica; (3) fundamenta- se na análise e na medida de todos os tempos executados num trabalho, visando eliminar os esforços desnecessários. (DUARTE, 2015, p. 22)

$\mathrm{O}$ desenvolvimento do método preferido ou ideal parte primeiramente da definição de um problema, onde são criados relatórios com base nas metas e objetivos existentes. Consequentemente será realizado a análise de problemas descrevendo todos os métodos incluindo especificações e restrições de produção. Desta forma, com o surgimento de possíveis soluções, são realizadas avaliações objetivando o método que proporciona menor custo e assim menor investimento, contribuindo para um processo de produção mais rápido e eficaz. Com a utilização desta metodologia torna-se possível processar uma sequência de operações que se aproximam do "ideal".

\subsection{Determinação do número de cronometragens}

Pinheiro et al. (2012, apud Martins e Laugeni, 2006), alega que a forma mais adequada de se definir o número de cronometragens ou ciclos $\mathrm{N}$ é determinado pela equação:

$$
\mathrm{n}=\left(\frac{\mathrm{Z} * \mathrm{R}}{\mathrm{Er}^{*} \mathrm{~d} 2 * \mathrm{xm}}\right)^{2}
$$

Sendo:

Z: Nível de Confiança da distribuição normal (geralmente utilizada entre (90 à 95\%) R: Amplitude da Amostra.

Er: É o Erro relativo, que depende do nível de confiança adotado.

d2: Coeficiente obtido na tabela de estatística específica que está em função da cronometragem realizada.

xm: Média de amostra.

3.4 Limite superior e inferior de controle

De acordo com Rosario et al. (2015, apud Peinado e Graeml, 2007), é preciso identificar se os valores (das amplitudes e das médias) de um fragmento estudado estão dentro dos limites determinados. Por essa razão, existem dois gráficos de controle que são utilizados mediante o limite Inferior (LIC) e o limite superior (LSC). Esses gráficos são os de amplitude e das médias.

Ainda conforme o autor, o gráfico de controle da média é representado pelas fórmulas:

$$
\mathrm{LSC}=\bar{x}+\mathrm{A} * \mathrm{R}(1) \mathrm{LIC}=\bar{x}-\mathrm{A} * \mathrm{R}(2)
$$

No qual:

$\bar{x}$ : Média dos tempos médios da amostra.

A: Coeficiente tabelado obtido através de cronometragens. R: Média das amplitudes da amostra.

E o gráfico das amplitudes é representado pela seguinte equação: 
$\mathrm{LSC}=\mathrm{D} 4 * \mathrm{R}(3)$

$\mathrm{LIC}=\mathrm{D} 3 * \mathrm{R}(4)$

Sendo assim:

D3 e D4 são coeficientes tabelados determinados a partir das cronometragens realizadas.

R: Média das amplitudes da amostra.

\subsection{Determinação do tempo médio}

De acordo com Rosario et al. (2015, apud PEINADO e GRAEML, 2007), dividir cada elemento das atividades com suas devidas cronometragens, somar os tempos de cada elemento e dividir pelas cronometragens obtidas, é uma forma eficiente de determinar o tempo médio do processo de produção.

3.6 Fatores de ritmo

Conforme Pinheiro et al. (2012), a análise é feita para verificar se o operário apresenta uma conduta sem muitas variabilidades ao exercer uma atividade. Verificar que o operador tem baixa variabilidade é de suma importância quando se realiza o estudo de tempos e movimentos, após a escolha de um operário muito abaixo ou acima da média pode ocorrer resultado que divergem da realidade do ambiente de investigação.

Foi selecionado um operário para o estudo e realizamos a avaliação de ritmo. Foi escolhido o estudo por distribuição de 52 cartas. Definindo o ritmo V do operário, pela fórmula:

Segundo Machado (2012), a velocidade "V" do operário é definida por meio

$$
V=\frac{\text { tempo internacional }(30 s)}{\text { tempo médio do funcionário }}
$$

de cronometragens, que tem como função denominar a velocidade normal de operação, no qual, é atribuído o valor 100 (ou 100\%). Para que não ocorra erros eventualmente a avaliação sistemática e contínuo dos cronometristas, usando operações padronizadas.

3.7 Determinação do tempo normal (TN)

De acordo com Rosario et al. (2015, apud Peinado e Graeml, 2007), afirma que é o tempo fundamental para a execução da atividade considerando-o a velocidade do operário. Desta maneira, é necessário primeiramente conhecermos a velocidade para que então o produto desta velocidade em conjunto com o tempo médio determine o tempo normal, conforme a fórmula a seguir definida pelos autores citados anteriormente:

$\mathrm{TN}=\mathrm{TM} * \mathrm{~V}$

\subsection{Capacidade produtiva}

Slack (2009) cita que a capacidade produtiva estabelece o potencial produtivo de uma empresa, retrata o objetivo ideal de produção de bens ou serviços que uma empresa pode realizar. Esse objetivo ideal representa um nível em que permite o máximo de lucro e o menor custo de produção, manutenção, mão-de-obra, etc. A formula para definição da capacidade produtiva, segundo Rosario et al. (2012, apud Peinado e Graeml, 2007), diante do tempo padrão (TP) e a carga horária de trabalho diário (TD):

$\mathrm{CP}=$ 


\subsection{Fator de tolerância}

Segundo Souza (2012, apud Silva e Coimbra, 1980), o tempo normal não possui tolerância, é simplesmente o tempo que é gasto para que um operário qualificado exerça uma atividade, trabalhando em ritmo normal. Por toda via, um operador não consegue exercer o trabalho o dia todo sem pausas.

4 DISCUSSÕES

\subsection{Descrição do processo}

Foi escolhido um funcionário aleatoriamente como objeto de estudo, pois se trata de uma amotragem aleatória simples, no qual, a partir de sua velocidade fosse definida a velocidade operária. Utilizou-se uma operação padronizada para verificar a eficiência deste funcionário.

Para a análise, foi escolhida a bombona plástica de cinco litros do xarope de guaraná, no qual, a etapa escolhida foi a embalagem e separação de estoque, notando que os processos que a procedem possuem tempo padronizado e uma participação considerável de máquinas, o que impediria cronometragens exatas em função da força humana, consequentemente no aperfeiçoamento dos funcionários na produção.

A primeira operação é feita pelo funcionário que constantemente busca as bombonas vazias para a realização da etapa seguinte, após isso, as bombonas são colocadas em cima da mesa e o funcionário as preenchem pelas 4 tubulações, no qual, sai o produto final. A terceira atividade constitui no funcionário realizar o fechamento da bombona que acaba de ser preenchida. A quarta atividade se baseia no agrupamento de 64 bombonas em cima da palheteia que em seguida é realizada a quinta atividade que é transportar e guardar no estoque as bombonas.

Com a identificação das atividades no qual trabalhou, foram realizadas 10 cronometragens em um total de 7 dias, no final de cada dia realizamos um cálculo de média do dia, no qual foi realizada somente sobre o funcionário selecionado para não haver erros eventuais.

\subsection{Cálculo do processo}

Primeiramente foi escolhido um funcionário aleatório como objeto de estudo, pois a escolha indefere o resultado já que posteriormente foi usado o cálculo para obtenção da eficiência deste funcionário, a partir de então realizou-se a atividade de Barnes (1977) como fator de ritmo para analisar a velocidade do operador. Para isso foram distribuídas 52 cartas em quatro pilhas, no qual, o tempo de distribuição segundo o autor é definida por 30s, então realizou-se uma regra simples de três para determinar a velocidade do operador, na qual foram gastos 29,06 segundos, sua velocidade é definida a seguir:

$$
\begin{aligned}
& 2906 \\
& V=- \\
& 30 \\
& V=96,87 \%
\end{aligned}
$$

Com o valor da velocidade de um tempo de tolerância de 10 minutos concedidos aos funcionários, foi elaborado uma tabela, mostrando as cinco atividades realizadas do A3, cronometradas dez vezes, por sete dias diferentes, em segundos. Entretanto, os valores encontrados poderiam ser descartados a qualquer momento se os valores médios e amplitudes dos dados coletados não se encaixasse nos níveis aceitáveis do controle estatístico, seriam necessárias novas realizações de cronometragens. 
Tabela 1: Atividades e Tempos gasto

\begin{tabular}{|c|c|c|c|c|c|c|c|c|}
\hline Atividades & $1^{\circ} \mathrm{T}$ & $\begin{array}{l}2^{\circ} \\
\mathrm{T}\end{array}$ & $3^{\circ} \mathrm{T}$ & $4^{\circ} \mathrm{T}$ & $5^{\circ} \mathrm{T}$ & $6^{\circ} \mathrm{T}$ & $\begin{array}{l}7^{\circ} \\
\mathrm{T}\end{array}$ & $\begin{array}{l}\text { Ritm } \\
\text { o }\end{array}$ \\
\hline $\begin{array}{l}\text { Busca de bombonas } \\
\text { vazias }\end{array}$ & $\begin{array}{l}15,9 \\
0\end{array}$ & $\begin{array}{l}16 \\
, 1 \\
0\end{array}$ & $\begin{array}{l}15,6 \\
3\end{array}$ & 16,07 & $\begin{array}{l}16,0 \\
1\end{array}$ & $\begin{array}{l}15,9 \\
3\end{array}$ & $\begin{array}{l}15 \\
8 \\
5\end{array}$ & $\begin{array}{l}96,87 \\
\%\end{array}$ \\
\hline $\begin{array}{l}\text { Preenchimento das } \\
\text { bombonas }\end{array}$ & 3,78 & $\begin{array}{l}4, \\
15\end{array}$ & 3,95 & 4,12 & 4,05 & 4,30 & $\begin{array}{l}3, \\
92\end{array}$ & $\begin{array}{l}96,87 \\
\%\end{array}$ \\
\hline $\begin{array}{l}\text { Fechamento } \\
\text { as } \\
\text { bombonas }\end{array}$ & 3,93 & $\begin{array}{l}3, \\
87\end{array}$ & 4,54 & 4,23 & 3,98 & 4,15 & $\begin{array}{l}3, \\
86\end{array}$ & $\begin{array}{l}96,87 \\
\%\end{array}$ \\
\hline $\begin{array}{l}\text { Carregamento para } \\
\text { palheteia }\end{array}$ & $\begin{array}{l}128, \\
9 \\
8\end{array}$ & $\begin{array}{l}14 \\
5 \\
3 \\
2\end{array}$ & $\begin{array}{l}138 \\
9 \\
4\end{array}$ & $\begin{array}{l}140 \\
8 \\
4\end{array}$ & $\begin{array}{l}130 \\
7 \\
6\end{array}$ & $\begin{array}{l}126 \\
8 \\
7\end{array}$ & $\begin{array}{l}12 \\
9 \\
5 \\
6\end{array}$ & $\begin{array}{l}96,87 \\
\%\end{array}$ \\
\hline $\begin{array}{l}\text { Colocar as bombonas } \\
\text { no estoque }\end{array}$ & $\begin{array}{l}48,2 \\
0\end{array}$ & $\begin{array}{l}55 \\
, 6 \\
4\end{array}$ & $\begin{array}{l}50,7 \\
9\end{array}$ & 60,02 & $\begin{array}{l}50,6 \\
1\end{array}$ & $\begin{array}{l}57,3 \\
5\end{array}$ & $\begin{array}{l}50 \\
3 \\
2 \\
\end{array}$ & $\begin{array}{l}96,87 \\
\%\end{array}$ \\
\hline$\sum$ & $\begin{array}{l}200 \\
7 \\
9\end{array}$ & $\begin{array}{l}22 \\
5 \\
0 \\
8\end{array}$ & $\begin{array}{l}213 \\
8 \\
5\end{array}$ & $\begin{array}{l}225 \\
2 \\
8\end{array}$ & $\begin{array}{l}205 \\
4 \\
7\end{array}$ & $\begin{array}{l}208, \\
6 \\
0\end{array}$ & $\begin{array}{l}20 \\
3 \\
5 \\
1\end{array}$ & - \\
\hline Tempo Médio & \multicolumn{3}{|c|}{213,24} & \multicolumn{5}{|l|}{210,72} \\
\hline Amplitude & \multicolumn{3}{|l|}{24,29} & \multicolumn{5}{|l|}{21,77} \\
\hline
\end{tabular}

Fonte: Autor. Ano 2017

Em seguida realizou-se o cálculo de cronometragens para determinar quantas deveram ser feitas, no qual, utilizou-se o nível de confiança de $95 \%(Z=1,96)$ e o valor tabelado de D2 para a amostra igual 2,709. Além disso, foi necessário encontrar o Tempo Médio e Amplitude das 7 cronometragens. Conforme as contas a seguir:

Cálculo da Média:

$x m=\underline{200,79+225,08+213,85+225,28+205,47+208,6+203,51}$

$\mathrm{xm}=211,8$

Cálculo de Amplitude:

$\mathrm{R}=225,28-200,79$

$\mathrm{R}=24,49$

Número de Cronometragens:

$\mathrm{n}=\left(\frac{1,96 * 24,49}{0,05 * 2,709 * 211,8}\right)^{2}$

$\mathrm{n}=2,799$ 
Visto que estava tudo de acordo, prosseguiu-se os estudos, o próximo passo foi a realização do cálculo do Tempo Médio para que posteriormente ser possível realizar o cálculo do Tempo Normal.

$$
\begin{aligned}
& T M 1=\frac{15,9+16,1+15,63+16,07+16,01+15,93+15,87}{7}=15,93 \\
& T M 2=\frac{3,78+4,15+3,95+4,12+4,05+4,3+3,92}{7}=4,04 \\
& T M 3=\frac{3,93+3,87+4,54+4,23+3,98+4,15+3,86}{7}=4,08 \\
& T M 4=\frac{128,98+145,32+138,94+140,84+130,76+126,87+129,56}{7}=134,46
\end{aligned}
$$

Com o tempo médio definido em cada atividade realizada é possível calcular o Tempo Normal destas, levando em consideração a velocidade do operário, de acordo com a atividade realizada no início do estudo. Desta forma foram realizou-se os seguintes cálculos:

$$
\begin{aligned}
& \text { TN1 }=15,93 * 96,87 \%=15,43 \\
& \text { TN2 }=4,04 * 0,9687=3,91 \\
& \text { TN3 }=4,08 * 0,9687=3,95 \\
& \text { TN4 }=134,46 * 0,9687=130,26 \\
& \text { TN5 }=53,28 * 0,9687=51,61
\end{aligned}
$$

Considerando que os valores acima pertencem a cada uma das atividades realizadas, em outras palavras, o Tempo Normal destas, a realização do somatório disponibilizará o tempo de operação da embalagem e separação de estoque do A3.

TNoperação $=$ TN1 + TN2 + TN3 + TN4 + TN5

TNoperação $=205,16$

Com os valores do Tempo Normal definido, foi possível realizar os cálculos para a obtenção do Tempo Padrão que cada operário leva para a realização de sua atividade, foi acrescentado o fator tolerância que são destinados para o mesmo praticar suas necessidades pessoais.

Fator de Tolerância:

$$
\begin{aligned}
& T P=T N * F T \\
& T P=205,16 *(1+0,18) \\
& T P=242,09
\end{aligned}
$$

Com os valores do Tempo Padrão definida é possível realizar os cálculos da capacidade produtiva de cada operário ao executar sua determinada atividade, considerando que a jornada de trabalho sejam oito horas.

$$
\begin{aligned}
& \mathrm{CP}=\frac{\mathrm{TD}}{T P} \\
& \mathrm{CP}=\frac{480 \text { minutos }}{242,09 \text { segundos }} \\
& \mathrm{CP}=\frac{480 \text { minutos }}{4,03 \text { minutos }}
\end{aligned}
$$


$\mathrm{CP}=191,1$

Conclui-se que a capacidade produtiva de cada operário é de 119 bombonas de A3 por dia.

Realizou-se a análise das cartas de controle, foi montado os gráficos de controle das médias e das amplitudes, é indispensável para a realização dos estudos, no qual, identificará se os valores estão dentro dos padrões, as amostras foram dividas (como demostrado na tabela 1), dessa forma foram determinados dois valores médios, onde XM1 é o resultante das três primeiras amostras e XM2 das outras quatro respectivamente.

$X M 1=213,24$

$X M 2=210,72$

$\overline{\bar{X}}=\frac{213,24+210,72}{2}=211,98$

Em seguida realizou-se os cálculos das amplitudes:

$R 1=24,29$

$R 2=21,77$

$R M=\frac{24,29+21,77}{2}=23,03$

Com os valores definido, foi realizado os cálculos dos limites superiores e inferiores do gráfico das médias:

$L S C=\overline{\bar{X}}+A * R M=211,98+(0,419 * 23,03)=221,63$

$L I C=\overline{\bar{X}}-A * R M=211,98-(0,419 * 23,03)=202,40$

No qual, A é determinado e igual a 0,419 referente ao número de cronometragens igual a sete, de acordo com a apêndice 1 .

Representa-se os valores obtidos através do gráfico, como demostra a figura 2.

Figura 2: Gráfico Das Médias

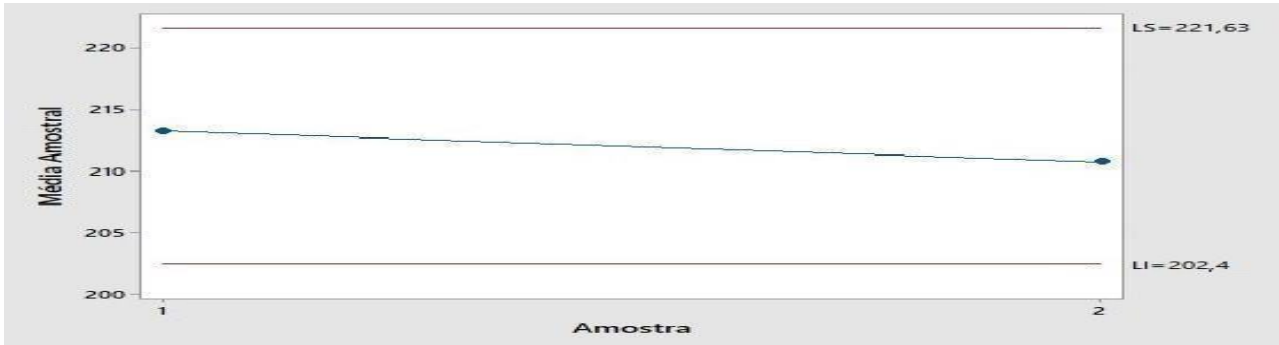

A seguir foi necessário realizar os cálculos para a obtenção do controle de amplitude, visando que D3 e D4 é um valor determinado pelo número de sete 
cronometragens, analisado pelo apêndice 1. No após a determinação dos valores realizamos a representação gráfica.

$$
\begin{aligned}
& \mathrm{LSC}=\mathrm{D} 4 * \mathrm{RM}=1,924 * 23,03=44,31 \\
& \mathrm{LIC}=\mathrm{D} 3 * \mathrm{RM}=0,076 * 23,03=1,75
\end{aligned}
$$

Figura 3: Gráfico de Controle Das Amplitudes

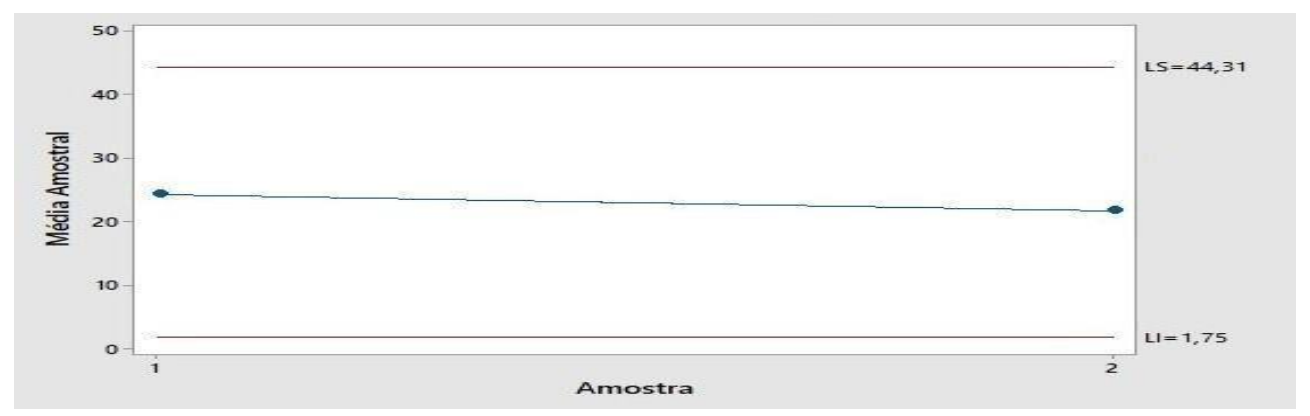

Fonte: Autor. Ano 2017

Sabendo-se que ambos valores se encontram dentro dos limites de controle, a amostra se encontra dentro dos parâmetros do controle estatístico, e visto isto, os sete valores se encontram validos, não necessitando descarta-los para a realização de novas cronometragens para o estudo.

\section{CONCLUSÃO}

A conclusão de que o estudo de tempo observado a partir dos dados cronometrados, possui um tempo padrão de quatro minutos, com fator de tolerância igual a 1.18. Tendo em mente que a carga horária da empresa é de oito horas, a capacidade produtiva foi estimada em 119 bombonas de A3 por operário em um dia de trabalho normal.

Alguns aspectos foram analisados durante a coleta de dados que estariam afetando um aumento do tempo realizado das operações, tais como, má distribuição das máquina no espaço, um pequeno congestionamento de pessoas em um determinado corredor o que acarretava atraso na chegada de produtos, além de uma distância considerável entre o produto destinado deve chegar para ser processado e o ponto de partida, e uma boa tarde do tempo era gasto com os funcionários deixando o setor de serrivços para carga e descarga de produtos. Foi observado que em determinados meses do ano alguns operários podem ser remanejados para outros setores, já que a demanda diminui em determinas estações do ano.

Deste modo, o estudo de tempo e movimento foi necessário para estimarmos a capacidade produtiva da fábrica, e também para o observamos de fatores que está diretamente ligado a estes cálculos. Esses resultados dos estudos mais afundo acerca das outras operações, podem ocasionar a resultados mais concretos e a melhorias significativas no tempo de produção, interferindo na produtividade.

\section{REFERÊNCIAS BIBLIOGRÁFICAS}

BRASIL. Decreto n. 6.871, de 4 de junho de 2009. Dispõe sobre a padronização, a classificação, o registro, a inspeção, a produção e a fiscalização de bebidas. Diário Oficial [da] República $\begin{array}{lllll}\text { Federativa do } & \text { Brasil, } & \text { Brasília, DF, } 05 & \text { jun. } & 2009 .\end{array}$

Disponível em: < $\quad$ http://www.agricultura.gov.br/assuntos/vigilancia- 
agropecuaria/ivegetal/bebidas-arquivos/decreto-no-6Acessado em 4 de maio de 2019.

BORTOLI, Henrique Weber. Aplicação da Cronoanálise para Melhoria do Processo de Suprimento da Linha de Montagem de uma Empresa de Grande Porte do Ramo Agrícola, 2013. p.13. In: BARNES, R.M. Estudo de tempos e movimentos: projeto e medida do trabalho.

CAMARGO, Mônica Cristiane Rojo et al. Determinação de hidrocarbonetos policíclicos aromáticos (HPAS) em guaraná em pó (Paullinia cupana). Ciência e Tecnologia de Alimentos, Campinas, v. 26, n.1, $2006 . \quad$ Disponível em:

<http://www.scielo.br/scielo.php?pid=S0101- 20612006000100036\&script=sci_arttext $>$. Acessado em 4 de maio de 2019.

Citisystems. Tabela 2: Tabela contendo as constantes (A2, A3, B3, B4, D3, D4). 2016. Disponível em: <https://www.citisystems.com.br/cartas-de-controle/>. Acessado em 27 de junho de 2017.

DUART, Geraldo. Dicionário de Administração e Negócios. P .22. Disponível em: $<$ https://books.google.com.br/books?id=s48Wk4Nj5EoC\&pg=PT592\&dq=tempo+e+m ovimento \&hl=pt- $\quad \mathrm{BR} \& \mathrm{sa}=\mathrm{X} \&$ redir_esc $=\mathrm{y} \# \mathrm{v}=$ onepage $\& \mathrm{q}=$ tempo $\% 20 \mathrm{e} \% 20$ movimento $\& \mathrm{f}=$ false $>$. Acesso em: 5 de junho de 2017.

Folha de S. Paulo, Refrigerante perde gás na crise, e consumidor migra para suco em pó, 2016. Disponível em:

<http://www.m.folha.uol.com.br/amp/mercado/2016/05/1768920-refrigerante-perde- gas-na-crise-econsumidor-migra-para-suco-em-po.html>. Acesso em 19 de abril de 2017.

LIMA, Rafael, Introdução à Cronoanálise com Exemplo, 2016. Disponível em: <http://www.estudoadministracao.com.br/ler/16-11-2014-como-fazer-citacoesAcesso em 22 de abril de 2017.

internet/>

MACHADO, Marcio Cardoso. Gestão de Operações IV (módulo2). 2012. Disponível em:

<http://marciocmac.dominiotemporario.com/doc/GOIV_2Bim_12.pdf >. Acesso em 23 de abril de 2017.

OLIVEIRA, J. W. Sistema de Informação, 2009. Disponível em:

<https://xa.yimg.com/kq/groups/22755187/1481008806/name/Proc.Neg.Atividade.pdf.

> Acesso em: 7 de março de 2017.

PINHEIRO, et al. Estudo de tempos e movimentos para determinação da capacidade produtiva de uma empresa de processamento de frangos, 2012. In: MARTINS, PETRÔNIO GARCIA;

LAUGENI, FERNANDO PIERO. Administração

da produção. $2^{\circ}$ ed. São Paulo: Saraiva, 2006. Disponível em:

<http://www.abepro.org.br/biblioteca/enegep2012_tn_sto_157_920_20618.pdf >. Acesso em 23 de abril de 2017.

RIBEIRO, B.D.; COELHO, M.A.Z.; BARRETO, D.W. Obtenção de extratos de guaraná ricos em cafeína por processo enzimático e adsorção de taninos. Brazilian Journal of Food Technology, v. 15, n. 3, 2012.

ROSARIO, et al. Estudo de tempos aplicado no processo de Finalização da Produção em uma Fábrica de Açaí. 2015. In: PEINADO, J.; GRAEMI, A. Administração da Produção: Operação Industriais e de Serviços. Curitiba: UnicenP. 2007. Disponível em:

<http://www.abepro.org.br/biblioteca/TN_STO_206_220_27763.pdf>. Acesso em 23 de abril de 2017.

SLACK, Nigel et al. Administração da produção. São Paulo: Atlas, 2009. Projeto Potencialidades Regionais Estudo de Viabilidade Econômica. Disponível em:

<http://www.suframa.gov.br/publicacoes/proj_pot_regionais/acai.pdf>. Aceso em 24 de abril de 2017. 
SOARES, Cláudio Magela. Controle Estatístico Aplicado ao Processo de Colheita Mecanizada de Milho.2016. In: MONTGOMERY, D.C. Introduction to Statistical Quality Control. New York: John Wiley, 1991. Disponível em:

<http://www.anais.ueg.br/index.php/cepe/article/view/7000/4604>. Acessado em 25 de Abril de 2017.

SOUZA JÚNIOR, A. G. Estudo Comparativo das Metodologias de Tempo Pré- determinadas MTM-UAS e BASIC-MOST - Aplicação Prática. 2010. Dissertação (Mestrado em Engenharia Mecânica em Ênfase em Produção) - Centro Universitário de FEI, São Bernardo do Campo, 2010.

SOUZA, Edson Luis de. Proposta e Aplicação de um Modelo de Cronoanálise para os Setores de Soldagem e Montagem de uma Empresa de Agronegócios, 2012. In: SILVA, A. V., COIMBRA, R.

R. C. Manual de Tempos e Métodos. São Paulo: Hemus, 1980. Disponível em:

<http://www.fahor.com.br/publicacoes/TFC/EngPro/2012/Edson_Luis_de_Souza.pdf>. Acesso em 24 de abril de 2017.

Apêndice 1: Tabela Contendo As Constantes

\begin{tabular}{|c|c|c|c|c|c|c|}
\hline & \multicolumn{2}{|c|}{ Médias } & \multicolumn{2}{c|}{ Desvio-padrão } & \multicolumn{2}{c|}{ Amplitudes } \\
\hline $\mathrm{n}$ & $\mathrm{A} 2$ & $\mathrm{~A} 3$ & $\mathrm{~B} 3$ & $\mathrm{~B} 4$ & $\mathrm{D} 3$ & $\mathrm{D} 4$ \\
\hline 2 & 1,88 & 2,659 & $\mathrm{O}$ & 3,267 & $\mathrm{O}$ & 3,267 \\
\hline 3 & 1,023 & 1,954 & $\mathrm{O}$ & 2,568 & $\mathrm{O}$ & 2,574 \\
\hline 4 & 1,729 & 1,628 & $\mathrm{O}$ & 2,266 & $\mathrm{O}$ & 2,282 \\
\hline 5 & 0,577 & 1,427 & $\mathrm{O}$ & 2,089 & $\mathrm{O}$ & 2,114 \\
\hline 6 & 0,483 & 1,287 & 0,03 & 1,97 & 0 & 2,004 \\
\hline 7 & 0,419 & 1,182 & 0,118 & 1,882 & 0,076 & 1,924 \\
\hline 8 & 0,373 & 1,099 & 0,185 & 1,815 & 0,136 & 0,864 \\
\hline 9 & 0,337 & 1,032 & 0,239 & 1,761 & 0,184 & 1,816 \\
\hline 10 & 0,308 & 0,975 & 0,284 & 1,716 & 0,223 & 1,777 \\
\hline 11 & 0,285 & 0,927 & 0,321 & 1,679 & 0,256 & 1,744 \\
\hline 12 & 0,266 & 0,888 & 0,354 & 1,646 & 0,283 & 1,717 \\
\hline 13 & 0,249 & 0,85 & 0,382 & 1,618 & 0,307 & 1,693 \\
\hline 14 & 0,235 & 0,817 & 0,406 & 1,594 & 0,328 & 1,672 \\
\hline 15 & 0,223 & 0,789 & 0,428 & 1,572 & 0,347 & 1,653 \\
\hline 16 & 0,212 & 0,763 & 0,448 & 1,552 & 0,363 & 1,637 \\
\hline 17 & 0,203 & 0,739 & 0,466 & 1,534 & 0,378 & 1,622 \\
\hline 18 & 0,194 & 0,718 & 0,482 & 1,518 & 0,391 & 1,608 \\
\hline 19 & 0,187 & 0,698 & 0,497 & 1,503 & 0,403 & 1,597 \\
\hline 20 & 0,18 & 0,68 & 0,51 & 1,49 & 0,415 & 1,585 \\
\hline 21 & 0,173 & 0,663 & 0,523 & 1,477 & 0,425 & 1,575 \\
\hline 22 & 0,167 & 0,647 & 0,534 & 1,466 & 0,434 & 1,566 \\
\hline 23 & 0,162 & 0,633 & 0,545 & 1,455 & 0,443 & 1,557 \\
\hline 24 & 0,157 & 0,619 & 0,555 & 1,445 & 0,451 & 1,548 \\
\hline 25 & 0,153 & 0,606 & 0,565 & 1,435 & 0,459 & 1,541 \\
\hline Fonte & Cis & & & & & \\
\hline
\end{tabular}

Fonte: Citisystems. Ano 2017 